\section{Generation of multiple ratio scales with a fixed stimulus attribute}

\author{
JOHN C. BAIRD, MICHAEL KREINDLER, and KENNETH JONES \\ Dartmouth College, Hanover, New Hampshire 03755
}

Psychophysical theories differ in the relative weight given to sensory and cognitive variables. Two opposing theories are described and tested in an experiment designed to vary a cognitive factor while maintaining a constant sensory factor. The method of magnitude estimation was used with the constant stimulus attribute of line length. The cognitive factor was varied by providing Ss with different feedback concerning the numerical values assigned to the largest and smallest lines in the series. This procedure led to multiple ratio scales for the same stimulus attribute. It is argued that these results support a theory which stresses both cognitive and sensory variables in the explanation of psychophysical functions.

For the past 15 years, our understanding of perception has been steadily advanced by results obtained with ratio methods. Ratio scales now exist for an impressive array of stimulus attributes covering most forms of sensory stimulation (Stevens, 1966). The psychophysical relation which describes these results is a power function of the type given in Eq. 1.

$$
\mathrm{R}=\alpha \mathrm{S}^{\mathrm{n}}
$$

Here $\mathrm{R}$ represents the S's response which depends upon the stimulus magnitude, $S$, raised to a power indicated by the exponent, n. Equation 1 shows the function in its simplest form, where the value of $n$ reflects the stimulus attribute under investigation (e.g., luminance, line length) and $\alpha$ is a multiplicative constant having to do only with the scale units employed. ' It is customary to determine the parameters in the power function by dealing with the logarithnic form of Eq. 1:

$$
\log \mathrm{R}=\mathrm{n} \log \mathrm{S}+\log \alpha .
$$

The description of results according to Eqs. 1 and 2 is a well-established part of psychophysics, but the exponent is a matter of considerable debate.

There are two basic theories available. One stresses sensory mechanisms as determinants of the psychophysical function and its exponent. The second theory claims that the psychophysical function, as well as the value of the exponent, depends upon learning experiences (Warren \& Warren, 1963). Either theory could be deterministic or probabilistic, which in practice anounts to the relative concern with judgment

* This rescarch was supported in part by Grant 5 R01 MH 14379-02 from the U.S. Public Health Service. We thank Wilfred Dauphinais and Joel Foldman for their cheerful help in constructing the apparatus. variability. The deterministic approach treats variability as random (uninteresting) error from a true scale. The probabilistic approach encompasses a variety of scales, the theory.

If we consider two theories (sensory and learning) which can be either deterministic or probabilistic and which can be applied either to the form of the psychophysical function or to the exponent, we can formulate $2 \times 2 \times 2=8$ alternative models. In addition, we can conceive of intermediate models in which some aspect of the function or exponent is due to sensory mechanisms and the remainder is due to learning (Baird, 1970a, b; Curtis, Attneave, \& Harrington, 1968). For the purposes of this paper, two relevant models were selected because of their importance and clarity of opposing predictions. In addition, one of the models has already received considerable attention in this laboratory (Baird, 1970a, b), and the present study fits into this larger research program. The opposing models can be called "deterministic-sensory" (DS) and "probabilistic sensory learning" (PSL). These are ideal, general models used to provide a framework for discussion of our experiment.

\section{The Deterministic-Sensory Model (DS)}

The most stringent model attributes the power function and the exponent solely to the action of sensory mechanisms (Stevens, 1961, 1966; Bond \& Stevens, 1969). This model is deterministic inasmuch as a unique scale is assumed to exist for each stimulus attribute, and this scale isn't supposed to vary subsiantially from one $S$ to another. Individual differences are not treated very extensively, and variability is considered to be random error or the result of method bias.

The Probabilistic-Sensory-Learning Model (PSL) and therefore treats variability as part of
According to the PSL model, psychophysical results are due both to sensory (perceptual) factors and to learning (cognitive) factors (Baird, 1970a, b). For example, it is suggested that the power function is due to the ratio instructions given in scaling experiments, whereas the exponent in Eq. 1 is viewed as a ratio between the perceptual and cognitive factors, as described in Eqs. 3 and 4:

$$
\begin{gathered}
\mathrm{R}=\alpha \mathrm{S}^{\mathrm{p} / \mathrm{c}} \\
\log \mathrm{R}=(\mathrm{p} / \mathrm{c}) \log \mathrm{S}+\log \alpha
\end{gathered}
$$

Variability can enter into the perceptual or cognitive parameters given in Eq. 3, and in future developments variability parameters should be incorporated more directly into this function.

At present it is not possible to decide between the DS and PSL models on the basis of data obtained for different stimulus attributes. According to the DS model, changes in attribute are associated with changes in the magnitude of the exponent in Eq. 1. Similarly, the PSL model claims that changes in stimulus attribute are reflected in the value of the perceptual factor $(\mathrm{p})$, while the cognitive factor (c) remains constant. Therefore, the determination of ratio scales for many different attributes will not assist us in choosing between the two models.

The purpose of the experiment reported here was to obtain data which would allow us to decide between the DS and PSL models for a particular experimental arrangement. A situation was designed so that the cognitive parameter was varied while the perceptual parameter was held constant (in Eq. 3). This was accomplished by using the same stimulus attribute (line length) with different values of the cognitive parameter. The PSL model predicts that these changes will produce specifiable changes in psychophysical judgments. On the other hand, the DS model would have great difficulty with this case, since no provision is made for variations in the cognitive factor (i.e., the DS model would predict the same exponent, regardless of the value of $c$ in Eq. 3, or the model simply would not apply).

The experimental arrangement also allowed us to study the basis of the power function and its dependence upon instructions to the $S$, independent of sensory operations associated with a fixed stimulus attribute.

\section{Subjects \\ METHOD \\ The participants in this experiment were 80 male undergraduates enrolled in an} introductory psychology course at 


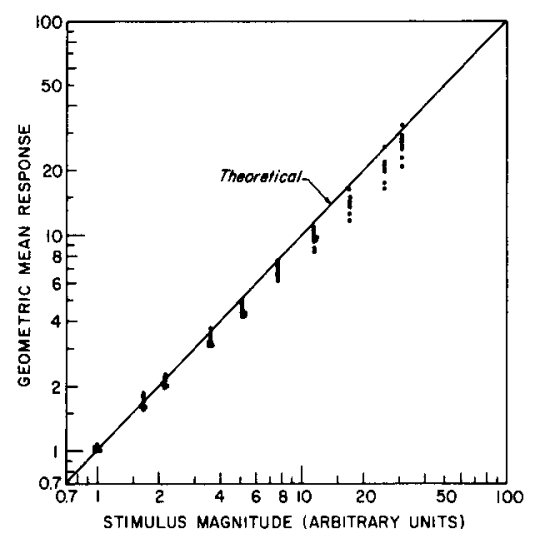

Fig. 1. The geometric mean of responses as a function of stimulus magnitude. Coordinates are logarithmic. Data are based upon magnitude estimates of line length in Phase 1. Each point on the graph is the geometric mean of $\mathbf{5 0}$ estimates obtained from $10 \mathrm{Ss}$. Eight groups (10 Ss in each) were run in the experiment, but group identity has not been maintained on the graph. The theoretical line represents an accurate match of responses and physical lengths. For more details, see the text.

Dartmouth College. Each S stated he had normal (corrected, if necessary) vision.

\section{Apparatus}

The $\mathrm{S}$ sat on an adjustable chair with his head in a large box $(8 \times 3 \times 2 \mathrm{ft})$ and viewed lines which were individually projected onto a screen at the opposite end. A voice microphone in the box was connected to a small amplifier and to a speaker which enabled $E$ to hear the verbal responses of $S$. The box also provided for natural ventilation and contained partial soundproof padding. The inside of the box was painted black. Viewing was binocular with relatively free head and eye movement.

Ten line lengths were employed (each of $1 / 4$-in. width): $.81,1.38,1.75,2.94,4.22$, $6.25,9.44,14,20.69$, and 25.12 in. Duplicate slides of each line length were made in order to facilitate multiple presentations. The slides were projected onto a transparent screen constituting one end of the box and located $6 \mathrm{ft}$ from $S$ 's eyes. The screen $(32 \times 20$ in.) was situated $91 / 2 \mathrm{ft}$ from the lens of a Carousel projector. The lines were presented at S's eye level in a horizontal orientation to the floor.

\section{Procedure}

The Ss were separated into eight groups, each consisting of 10 individuals. The experiment consisted of two phases. Phase 1 was a control for the manipulations introduced in Phase 2.

Phase 1 employed a procedure of magnitude estimation. The smallest line in the series was designated as a standard and was assigned the arbitrary value of 1 -unit length. The $S$ was instructed to assign numbers to the lengths of the comparison lines according to their judged ratio in respect to the standard. The order of stimulus presentation was randomized in blocks of 10 ; that is, all the lines were seen once before moving on to the next series. All $S s$ in a group received the same stimulus orders, which were constant across groups. Each $S$ made five judgments of each line (presented individually), including the standard, which was part of the comparison series. Exposure time was 8 sec per stimulus.

Phase 2 consisted of an experimental manipulation in which some groups were given feedback concerning the numerical value assigned to the largest line in the series. The smallest line was again designated as 1 unit for all Ss in all groups, and as before, the procedure consisted of five blocks of 10 randomized stimuli. However, the largest line in the series was also assigned a numerical value (by $E$ ) for $\mathrm{Ss}$ in seven groups. Different groups of Ss were told different numerical values for the largest standard. The specific values were derived from Eq. 3, in which the exponent of the power function is comprised of two basic factors, $p$ and c. In Phase 2 we attempted to effect changes in $c$ by assigning a numerical value to the largest line. This procedure will be explained in more detail in the following section.

Several assumptions were made in order to generate values for the largest standard. In previous work (Baird, 1970b), it was found that 4.6 was a reasonable estimate of c, but that a slightly smaller value, for example, 4.0 , led to more accurate predictions of empirical exponents. Consequently, in this study we assumed that the cognitive factor was 4.0 when $S$ were not given information concerning the value of more than one stimulus in the series (Phase 1). If the exponent for length is 1.0 , and $c=4.0$, then $p$ must be 4.0 . The selected theoretical values of $\mathrm{c}$ for Phase 2 were $1.33,2.0,3.0,4.0,5.33,8.0$, and 12 . Assuming that the perceptual factor $(p)$ is 4.0 , the exponents generated by Eq. 3 are $3.0,2.0,1.33,1.00, .75, .50$, and .33 , respectively.

Next, we determined the theoretical value for the largest stimulus in the series for each of the exponents above. From the highest to the lowest exponent, the values for the largest stimulus in the series were $29,577,956.24,97.06,30.92,13.11,5.56$, and 3.14. These predictions are based on the assumption that the smallest stimulus is normalized to l-unit length.

Each of seven groups was assigned one of the values for the largest stimulus. The eighth group was a control and received the same instructions as in Phase 1 . Note that the group with $c=4.0$ received accurate feedback on the size of the largest stimulus. That is, the ratio between the two standards was the actual physical ratio of the largest to the smallest stimulus.

The Ss in the experimental groups were told they would receive information concerning the largest stimulus and that this information need not represent the actual physical ratios existing between the largest and smallest lines. They were told that $E$ had a certain relationship in mind between the line lengths. Feedback for the largest line was information to aid them in determining this relationship. It was their task to assign numbers to the lines in the series which seemed appropriate, given their knowledge of the values of the largest and smallest lines. The two standards were presented and identified by their numerical values before each set of 10 trials. The entire experiment required approximately $35 \mathrm{~min}$ for each $\mathrm{S}$.

To summarize: the purpose of the experimental manipulation was to alter Ss' conception of their sensitivity to line-length ratios. The minimum number of standards needed to accomplish this is two. We chose the two end stimuli for this purpose. The Ss were not deceived but were instructed to judge lengths in accord with the sample values assigned to the smallest and largest stimuli. Primarily, we wanted to find out if Ss would generate power functions under these conditions, and if so, whether or not the exponent of such a function would agree with theoretical predictions.

\section{RESULTS AND DISCUSSION}

Standard analyses were employed to compare the results of Phases 1 and 2 .

\section{Phase 1 (Control)}

Preliminary analysis showed no significant differences among judgments on the five trials. Consequently, the geometric mean was computed for each group's judgments of each stimulus magnitude. In Fig. 1, the log of the geometric mean is plotted as a function of the log stimulus magnitude in order to determine the linearity of the data when presented in the form of Eq. 2. The groups were not individually designated on the graph because of the close agreement of the points.

The method of least squares was used to determine the slope and $y$-intercept of the linear function for each of the eight groups and for the combined data from all groups. There is reasonable agreement among the groups (see Table 1), with most exponents 
Table 1

Summary of Results for Phase 1 (Control)

\begin{tabular}{cccccccc}
\hline Group & Exponent & $\begin{array}{c}y \text {-Intercept } \\
(\log 10 \alpha)\end{array}$ & $\mathrm{r}^{2}$ & $\begin{array}{c}\text { Theoretical } \\
\mathrm{t}^{*}\end{array}$ & $\begin{array}{c}(1.0) \\
\mathrm{p}\end{array}$ & $\begin{array}{c}\text { Empirical } \\
\mathrm{t}^{*}\end{array}$ & $\begin{array}{c}(.93) \\
\mathrm{p}\end{array}$ \\
\hline 1 & .93 & -.0023 & .999 & 2.205 & $<.10$ & .017 & n.s. \\
2 & .89 & .0002 & .998 & 2.753 & $<.10$ & .960 & n.s. \\
3 & .94 & .0370 & .999 & 2.224 & $<.10$ & -.219 & n.s. \\
4 & .86 & .0237 & .999 & 4.156 & $<.10$ & 2.103 & $<.10$ \\
5 & .96 & -.0142 & .999 & 1.406 & n.s. & -.940 & n.s. \\
6 & .91 & .0375 & .998 & 2.070 & $<.10$ & .443 & n.s. \\
7 & .95 & -.0128 & .998 & 1.588 & n.s. & .771 & n.s. \\
8 & 1.02 & -.0220 & .998 & -.589 & n.s. & 2.965 & $<.10$ \\
All Ss & .93 & -.0059 & & & & & \\
\hline
\end{tabular}

${ }^{*}$ Two-tailed $t$ test, $9 d f$.

being somewhat less than 1.0. The $y$-intercepts are negligibly small (antilog equals approximately 1.0), and the coefficients of determination $\left(\mathrm{r}^{2}\right)$ given in Column 4 are exceptionally high, indicating that good fits were obtained. These results validate Eqs. 2 and 4. The exponent for the combined data from all groups is .93 and falls well within the boundaries of exponents expected for line length (Baird, 1970c). Group 4 has the lowest exponent, and Group 8 the highest. This type of result underlies one of the frustrations encountered in empirical research, since these two groups were controls for the manipulations introduced in Phase 2.

T tests are appropriate to compare exponents with the theoretical value of 1.0. Exponents based on geometric means obtained from individual $S$ s were used for these tests. In testing the significance of $t$ values a probability level of .10 was deemed reasonable, since rejection of the null hypothesis was not anticipated and, in view of our later use of these data as controls, we were a little concerned about the possibility of failing to reject the null hypothesis when in fact it was false. For somewhat analogous reasons, a stringent criterion for significance (.01) was used when differences were predicted on theoretical grounds (see Table 2). Results for five out of the eight groups were significantly different from the theoretical value of 1.0 , which verifies expectations based on Fig. 1, where there is a slight underestimation. It is possible that underestimation was due to the position of the standard at the end of the stimulus range (see Baird, 1970c).

The exponent obtained from all the data (combined groups) in Phase 1 served as a control value for Phase 2 . The original plan was to use 1.0 as a theoretical value, if possible, since this was the basis for deriving numerical values for the largest standard in Phase 2. Because underestimation occurred in Pliase 1, we adjusted the theoretical exponents for Phase 2 by a factor of .93 (Phase 1 control value). $T$ tests were performed on the data in Table 1 to test for differences between .93 and the exponent obtained for each group. The results of the tests are given in the last two columns of Table 1 , where it can be seen that only Groups 4 and 8 (with the highest and lowest exponents) are significantly different from 93 .

The variability of ratio judgments has an established characteristic for the attribute of line length. In previous work, the log relative error (standard deviation divided by the arithmetic mean) was a negatively accelerated function of the log stimulus magnitude (see Baird, 1970c). By calculating the same measures for the data in Phase 1, we checked on the consistency of our procedure with that used by other investigators. In Fig. 2 the $\log$ of the relative error is plotted as a function of the $\log$ of the stimulus magnitude for each of the eight groups. Each filled point in the the results. For more details, see the text and Fig. 1. figure is based upon 50 judgments, whereas the open circles represent 400 judgments obtained from all Ss. The smooth function through the points has been drawn to emphasize the general trend, which agrees very well with previous results.

\section{Phase 2 (Experimental)}

The analyses used in Phase 1 were also used in Phase 2. Geometric means were computed for each group, and these results are plotted in Fig. 2 in log-log coordinates. The method of least squares was used to fit straight lines to the data of each group. These straight lines appear in Fig. 2 for Groups 1 through 5 and Group 8. The two highest functions (Groups 6 and 7) are obviously not linear, and straight lines do not appear for these groups.

It should be recalled that, as the slope of a function increases, the value of $c$ decreases. The experimental manipulations in Groups 6 and 7 generated extremely large response ranges. This may be one contributing factor to the nonlinearity of the functions for these groups. Fairly smooth linear functions were obtained over the entire stimulus range for the remaining groups.

It is significant that the pattern formed by the functions in Fig. 3 closely resembles the patterns found when stimulus attributes are varied. In the present case, the stimulus attribute was fixed, but a cognitive factor was varied. Hence, it is

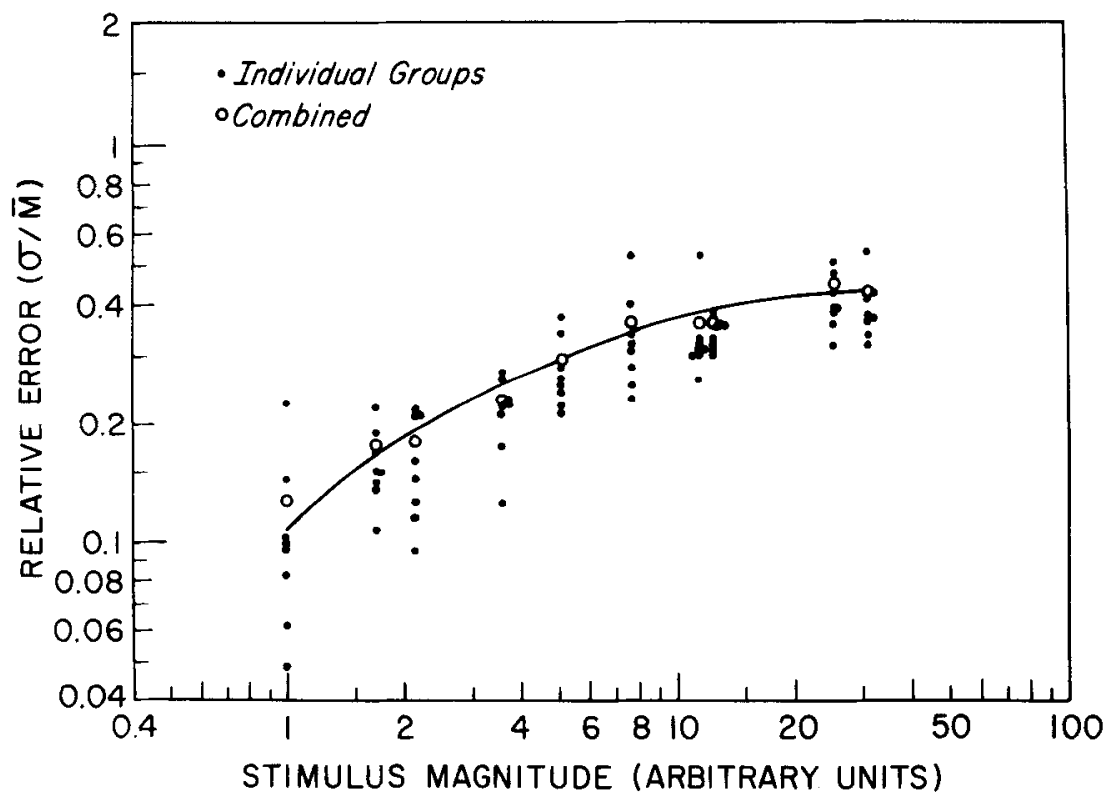

Fig. 2. The relative error (standard deviation divided by the mean) as a function of stimulus magnitude. Coordinates are logarithmic. Data are based upon magnitude estimates of line length in Phase 1. Each filled point on the graph is based upon 50 estimates obtained from 10 Ss. The open circles represent data based on the combined estimates (400) of all Ss. The smooth solid line has been drawn to emphasize the trend of 


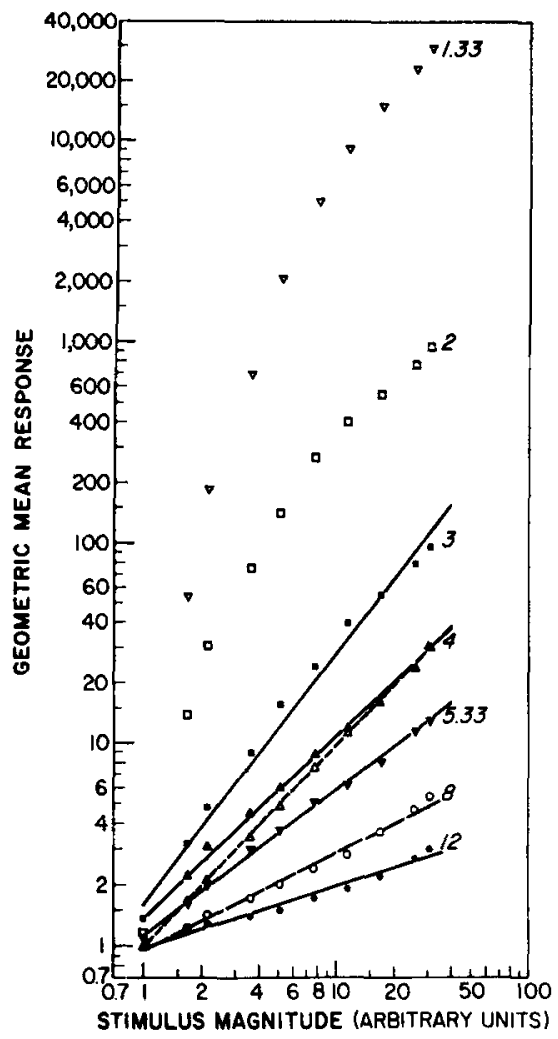

Fig. 3. The geometric mean of responses as a function of stimulus magnitude. Coordinates are logarithmic. Data are based upon magnitude estimates of line length in Phase 2. The numbers identifying the functions refer to the theoretical value of the cognitive factor (c) in Eq. 3. The value of $c$ was manipulated by altering the numerical value assigned to the largest stimulus (standard) in the series. The smallest stimulus served as a second standard and was assigned the value 1.0 for all groups. The two functions labeled $c=4$ are controls. The open triangles there are for Group 8. The filled triangles are for Group 4. The remaining groups can be identified from lowest to highest slopes as corresponding to Groups 1 through 7. For more details, see the text and Fig. 1 .

possible to produce the same changes in exponents by varying either the stimulus attribute (perceptual factor) or the cognitive factor.

An unexpected finding is that the $y$-intercept of the function also varies systematically with changes in $\mathrm{c}$ and, therefore, with changes in the exponent. As the exponent increases, the $y$-intercept increases, as can be seen in Fig. 3. Since the main concern here is with exponents, this result will not be pursued further at this time, except to note that the $y$-intercept seems to be positively correlated with the size of the response range.

Table 2

Summary of Results for Phase 2 (Experimental)

\begin{tabular}{|c|c|c|c|c|c|c|c|c|}
\hline \multirow[b]{2}{*}{ Group } & \multirow{2}{*}{ Exponent } & \multirow{2}{*}{$\begin{array}{l}y \text {-Intercept } \\
t\left(\log _{10} \alpha\right)\end{array}$} & \multirow[b]{2}{*}{$r^{2}$} & \multirow{2}{*}{$\begin{array}{c}\text { Theoretical } \\
\text { Exponent } \\
\text { (Adjusted) }\end{array}$} & \multicolumn{2}{|c|}{$\begin{array}{c}\text { Experimental } \\
\text { vs Control } \\
\end{array}$} & \multicolumn{2}{|c|}{$\begin{array}{l}\text { Experimental } \\
\text { vs Theoretical }\end{array}$} \\
\hline & & & & & $\mathrm{t}^{* *}$ & $\mathbf{p}$ & $\mathrm{t}^{* *}$ & $\mathrm{p}$ \\
\hline 1 & .31 & -.0134 & .977 & .31 & -20.40 & $<.01$ & -.25 & n.s. \\
\hline 2 & .48 & -.0216 & .990 & .46 & -10.00 & $<.01$ & -1.68 & n.s. \\
\hline 3 & .71 & .0488 & .997 & .70 & -7.16 & $<.01$ & -1.08 & n.s. \\
\hline 4 & .90 & .1316 & .994 & .93 & 1.92 & n.s. & 1.59 & n.s. \\
\hline 5 & 1.24 & .2173 & .983 & 1.24 & 9.94 & $<.01$ & .066 & n.s. \\
\hline 6 & 1.71 & .6841 & .903 & 1.86 & 8.01 & $<.01$ & 2.33 & $<.10$ \\
\hline 7 & 2.55 & 1.076 & .899 & 2.79 & 10.26 & $<.01$ & 1.51 & n.s. \\
\hline 8 (Control) & 1.00 & -.0131 & .999 & .93 & 1.01 & n.s. & -2.16 & $<.10$ \\
\hline
\end{tabular}

*Values given in the Method section adjusted by a factor of 93 . *Two-tailed $t$ test, 9 df.

Group exponents, y-intercepts, and coefficients of determination $\left(\mathrm{r}^{2}\right)$ are presented in Table 2. The fits of the straight lines are not quite as good as in Phase 1. This is especially noticeable for Groups 6 and 7.

A series of $t$ tests was performed to test for differences between empirical exponents obtained in Phases 1 and 2. The results are given in Table 2. A probability level of .01 was required for rejection of the null hypothesis. Highly significant differences were obtained for all the groups except for the two controls (Groups 4 and 8). This is as it should be. Group 4 received accurate feedback concerning the actual length of the largest line in the series. Group 8 received no feedback for the largest line. Hence the judgments of these two groups should not differ between Phases 1 and 2.

A second series of $t$ tests was performed between the experimental exponents (Phase 2) and the adjusted theoretical values (multiplied by .93) given in Column 5. The last two columns in Table 2 contain these results. Group 6 is the only one of the seven groups receiving experimental treatment which had an exponent significantly different from the theoretical (adjusted) exponent. Because of poor linear fits for Groups 6 and 7, apparent in Fig. 3, one cannot place too much weight on $t$ tests for these groups, but the overall trend is consistent with predictions of the PSL model.

The analysis of variability appears in Fig. 4, where the log of the relative error is plotted against the $\log$ of the stimulus magnitude for Groups 1 through 7. A consistent and unexpected pattern emerges in Fig. 4. When the value of $\mathrm{c}$ is very large, both the smallest and the largest stimulus magnitudes appear to serve as anchors for judgments. The relative error is low at the end points and maximum in the middle of the stimulus range. This particular result complicates the development of an adequate description of variability (cf Fig. 4 and Ekman \& Künnapas, 1969).

The unexpected part of the results can be described as follows: As the cognitive factor (c) decreases, the response range increases and the relative error increases. The largest stimulus in the series still appears to serve as an anchor, but the relative error for the smallest stimulus increases, as do the relative errors for adjacent stimulus magnitudes. We might offer several explanations for this unique variability function, but it would be pointless to do so at this stage without the benefit of more extensive data or theory.

\section{GENERAL DISCUSSION}

Two basic models have been proposed here to explain psychophysical results. The first is the deterministic-sensory (DS) model. According to this model, both the

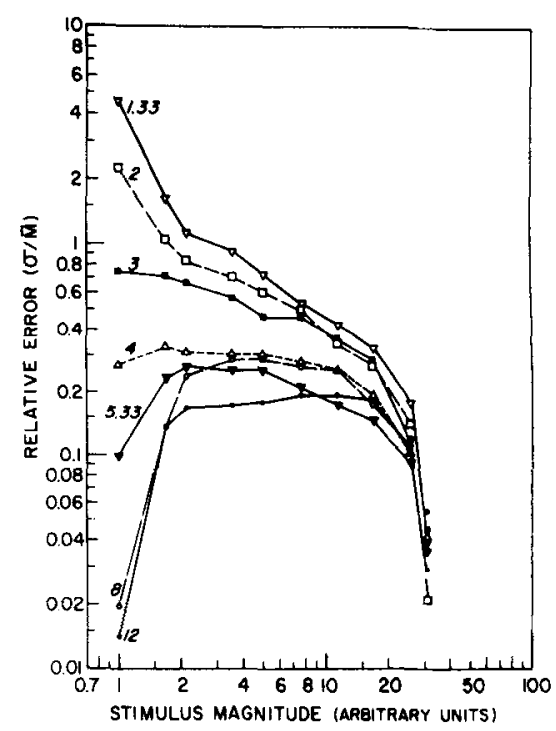

Fig. 4. The relative error as a function of stimulus magnitude. Coordinates are logarithmic. Data are based upon magnitude estimates of line length in Phase 2. The numbers identifying the functions refer to the theoretical value of the cognitive factor (c) in Eq. 3. Groups 1 through 7 correspond to the heights of the functions; i.e., for Group 1, $c=12$; for Group 7, $c=1.33$. For more details, see the text and Fig. 2. 
power function and its exponent are determined by sensory mechanisms which vary with the stimulus attribute. This model pays little attention to the variability of judgments, since it is assumed that a unique scale exists for each attribute, and that variance measures reflect random error around this true scale.

The DS model can be contrasted with a model of probabilistic sensory learning (PSL), which has a different conception of psychophysical results. According to this model, results are due to the interaction of a perceptual (sensory) and a cognitive (learning) factor.

A test of the applicability of the DS and PSL models was conducted in the present study by varying the cognitive factor while keeping the perceptual factor constant. The results of this investigation and their specific bearing on the two models will be discussed for three variables: the power function, the exponent, and the response variability.

\section{The Power Function}

If the power function were due solely to sensory mechanisms, it would be difficult to understand why Ss generated radically different ratio scales with the same stimulus attribute in Phase 2. It has been proposed by Baird $(1970 \mathrm{a}, \mathrm{b})$ that the power function is determined by the ratio instructions given to $S$. Different instructions (e.g., category estimation) produce different psychophysical functions. The instruction variable was held constant in the present study, and therefore, power functions were found both in Phases 1 and 2. This suggests rather strongly that the power function is not a unique characteristic of the sensory mechanism. These findings confirm predictions of the PSL model and are inconsistent with the DS model.

The foregoing statcments are tempered, however, by the fact that the power function collapses for high response ranges (Fig. 3). For these cases a linear function would hold over a small range of stimuli. It is interesting to note that small stimulus ranges are used for most conditions where the exponents are largc. Poulton (1968) has used such evidence to claim that the value of the exponent depends in part upon the range of stimulus magnitudes. The present investigation leads us to entertain an alternative hypothesis: When stimulus attributes are varied, the negative correlation between stimulus range and magnitude of the exponent is not a causative relationship. The reason for this correlation may be that Es proportionally reduce stimulus range with increases in the exponent in order to keep the response range within manageable limits. Therefore, the exponent really does reflect the responses of sensory mechanisms when the cognitive factor is kept relatively constant. This is in accord with both the DS and PSL models.

\section{Exponents}

We have shown that changes in exponents can be obtained by variation of a cognitive factor whose effect is similar to that found when stimulus attributes are varied. This finding supports the specific predictions of the PSL model, but cannot be handled by the DS model.

\section{Variability}

The variability for different cognitive values (Phase 2) was quite different from the variability obtained in the control condition (Phase 1). This is probably due to the use of anchors in the feedback conditions. If the cognitive factor could be changed without introducing an anchor effect, it should then be possible to obtain identical response variance by appropriate manipulation of either the perceptual or cognitive factor.

In summary, a choice between the DS and PSL models must be predicated upon the procedures under discussion. If the cognitive factor is held constant, the DS model will provide an adequate description of results. However, if one admits the existence of a cognitive variable, the PSL model is a better choice since it includes a parameter to handle this factor. Furthermore, if the cognitive factor is varied, the DS model is inapplicable. It remains to be seen whether or not the relationships found in the present study will occur for other stimulus attributes and for other procedures for manipulating the cognitive factor.

\section{REFERENCES}

BAIRD, J. C. A cognitive theory of psychophysics: I。 Information transmission, partitioning and Weber's Law. Scandinavian Journal of Psychology, 1970a, 11, 35-46.

BAIRD, J. C. A cognitive theory of psychophysics: II. Fechner's law and Stevens' law. Scandinavian Journal of Psychology, 1970b, 11, 89-102.

BAIRD, J. C. Psychophysical analysis of visual space, London: Pergamon, 1970c,

BAIRD, J. C., ROMER, D., \& STEIN, T. Test of a cognitive theory of psychophysics: Size discrimination. Perceptual \& Motor Skills. 1970, 30, 495-501.

BAIRD, J. C., \& STEIN, T. When power functions fail: A theoretical explanation. Perceptual \& Motor Skills, 1970, 30.415-425.

BOND, B., \& STEVENS, S. S. Cross-modality matching of brightness to loudness by five-yearolds. Perception \& Psychophysics, $1969.6,337-339$.

CURTIS, D. W., ATTNEAVE, F., \& HARRINGTON, T. L. A test of a two-stage model of magnitude judgment. Perception \& Psychophysics, 1968, 3, 25-31.

EKMAN, G.. \& KÜNNAPAS, T. Distribution function for similarity estimates. Perceptual \& Motor Skills, 1969, 29, 967-983.

MARKS, L. E.. \& STEVENS, J. C. The form of the psychophysical function near threshold. Perception \& Psychophysics. 1968, 4, 315-318.

POULTON, E. C. The new psychophysics: Six models for magnitude estimation. Psychological Bulletin, 1968, 69, 1-19.

STIEVENS, S. S. The psychophysics of sensory function. In W. A. Rosenblith (Ed.), Sensory communication. New York: Wiley, 1961.

STEVENS, S. S. The operation known as judgment, American Scientist, 1966, 54. 385401 .

WARREN, R, M., \& WARREN, R. P. A critique of S. S. Stevens' "New Psychophysics." Perceptual \& Motor Skills, 1963, 16. 797-810.

\section{NOTES}

1. Occasionally, constants are added or subtracted from the stimulus or response values in order to obtain a better fit to the data (sec Marks \& Stevens, 1969; Baird \& Stein, 1970).

(Accepted for publication November 16, 1970.) 